\title{
Réponse à Joëlle Aden : le corps à la lumière de l'émergentisme linguistique.
}

\section{Grégory Miras}

\section{(2) OpenEdition}

1 Journals

\section{Édition électronique}

URL : https://journals.openedition.org/rdlc/1442

DOI : $10.4000 /$ rdlc. 1442

ISSN : 1958-5772

Éditeur

ACEDLE

\section{Référence électronique}

Grégory Miras, "Réponse à Joëlle Aden : le corps à la lumière de l'émergentisme linguistique. », Recherches en didactique des langues et des cultures [En ligne], 14-1 | 2017, mis en ligne le 14 janvier 2017, consulté le 21 septembre 2021. URL : http://journals.openedition.org/rdlc/1442 ; DOI : https:// doi.org/10.4000/rdlc. 1442

Ce document a été généré automatiquement le 21 septembre 2021.

\section{c) (i) $९$}

Recherches en didactique des langues et des cultures is licensed under a Creative Commons AttributionNonCommercial-NoDerivatives 4.0 International License 


\title{
Réponse à Joëlle Aden : le corps à la lumière de l'émergentisme linguistique.
}

\author{
Grégory Miras
}

\section{Introduction}

1 Si pour certains « penser consiste entre autres à organiser ses représentations du monde en catégories cognitives" (Claverie, 2010:2), l'émergentisme constitue un cadre qui nécessite de concevoir ce qui est au-delà des éléments eux-mêmes. La catégorisation est l'un des fonctionnements humains les plus primitifs, mais au cœur de nos sociétés modernes; cette tendance, reliée au monde scientifique, s'est traduite en une vision reposant sur « un idéal ou des espoirs d'intelligibilité intrinsèque, fondés sur le postulat qu'une structure se suffit à elle-même et ne requiert pas [...] le recours à toutes sortes d'éléments étrangers à sa nature» (Piaget, 1968: 6). Les avancées technologiques de recherche en neurosciences de la cognition, depuis les années 90 , conduisent notamment vers une nouvelle conception des domaines du monde vivant. La didactique des languescultures n'échappe pas à cette tendance.

2 Nous chercherons à questionner l'article de Joëlle Aden (JA), et notamment son positionnement enactif, dans une perspective émergentiste. Nous tenterons d'analyser comment le corps et l'esprit peuvent être théorisés dans un tel cadre à travers une analyse « polythéiste » (Narcy-Combes \& Narcy-Combes, 2000 inspirés par Bourdieu, 1979 et Block, 1999) ou plurielle de la dualité corps-esprit. Nous terminerons en revenant sur les implications dans le champ de l'éducation, et plus particulièrement le domaine de la didactique des langues-cultures, proposées par JA. 


\section{Pour une approche plurielle des représentations corps-esprit}

3 La question du lien corps-esprit prend des formes différentes en fonction des domaines dans laquelle elle s'inscrit. On relèvera trois positions principales :

- Matérialiste : nous ne sommes qu'un organisme physique dont l'esprit est un épiphénomène (Simar, 2009 : 32-61) ;

- Idéaliste : nous ne sommes que conscience (Courville, 2009: 62-79);

- Dualiste : nous sommes le produit d'une interaction entre corps et esprit (Demars, 2009 : 9-31).

4 La question du dualisme corps-esprit porte souvent, comme le soulignent les trois positions mentionnées ci-dessus, sur les fondements théoriques et empiriques qui attesteraient ou, au contraire, invalideraient les liens entre ces deux notions. Cependant, une position méta amènerait à se questionner sur ce qui pousse les individus à trouver du dualisme dans le monde qui les entoure. Sur cette question, nous rejoignons le positionnement de JA qui va à l'encontre de l'idée selon laquelle «le monde que nous percevons serait indépendant du sujet qui le perçoit ». Comme le rappelle Claverie (2010: 2) : « [la pensée prototypique est] celle qui nous permet une conscience du monde à partir des groupes d'éléments que l'homme classe dans son système représentationnel ». Classer les notions et concepts de son environnement permet d'être capable d'en comprendre les fonctionnements en s'appuyant sur des représentations déjà connues. Une autre manière d'aborder ce processus cognitif, de compréhension de l'inconnu par le connu, est l'analogie. Pour Hofstadter \& Sander (2013), l'essence de la cognition humaine résiderait dans le processus d'analogie qui permettrait de comprendre la nouveauté sous le prisme de ce que l'on connait déjà. Ce processus rappelle celui d'assimilation ${ }^{1}$ décrit par Piaget (1964), d'obédience constructiviste, comme le souligne Narcy-Combes (2014). Dans le prolongement de cette réflexion sur les frontières cognitives, les disciplines elles-mêmes influencent la manière de traiter des notions comme le corps et l'esprit. Pour Morin (1990), l'hyperspécialisation isole la discipline par rapport aux autres et par rapport aux problèmes qui transcendent les disciplines et Claverie $(2010: 13)$ rajoute que :

penser la transdisciplinarité est donc une tâche instable [...]. La pensée transdisciplinaire est toujours en action, toujours en dépense d'énergie, toujours donc facile à abandonner d'autant qu'elle ne s'inscrit pas bien dans les espaces que nous réserve la société.

D'autres, comme Loty (2005), vont plus loin, en parlant d'« indisciplinarité » dont les enjeux seraient qu'il reviendrait «au chercheur singulier de définir son corpus et ses méthodes, et surtout le sens de sa recherche, avec les disciplines, contre les disciplines, ou hors discipline» (Ibid.: 258). Pour cet auteur, se positionner dans une discipline revient à ériger des frontières là où les processus complexes, en milieu écologique, n'en ont pas. Loty (Ibid.: 247) rejoint Morin lorsqu'il affirme que «le processus de disciplinarisation des savoirs, qui va de pair avec celui de spécialisation, est d'abord un processus de nature politique $»$.

Cette première partie nous a permis de faire émerger les tensions qui s'exercent autour de la question de la dualité corps-esprit. Ces tensions sont plurielles et complexes et elles se situent à la frontière de la psycho-cognition humaine et de l'épistémologie des sciences. Envisager une dualité, c'est avant tout se poser des questions sur la capacité 
humaine à envisager la complexité (Morin \& Lemoigne, 1999) et donc la possibilité que tout processus ne soit que continuum en dehors d'une « pensée binaire » (JA). Toutefois, à défaut de pouvoir statuer sur une position définitive sur le dualisme corps-esprit, nous suivrons, dans cette réflexion, le postulat des sciences cognitives, selon lesquelles, «les conduites et les processus mentaux sont sous-tendus par des événements physicochimiques ayant leur siège dans le cerveau » (Seron, 1998: 35). Dans cette perspective, Frith $(2010: 41)$ envisage la relation corps-esprit de manière réciproque tout en précisant que «la relation entre cerveau et esprit n'est pas parfaite. Il ne s'agit pas d'une correspondance point à point ». Dans ce sens, nous appuyons le positionnement de JA pour qui « [le monde et l'image que je me fais du monde] se définissent mutuellement». Afin de préciser ce positionnement, nous nous intéresserons à l'émergentisme en tant que cadre d'éclairage de ces questions du corps et de l'esprit.

\section{Emergentisme : une vision de la corporéité}

Dans son article, JA signale que "Varela [...] présente son paradigme comme une extension de l'émergentisme »; elle précise également que « la perspective enactive s'est développée à partir d'une constellation de théories des systèmes complexes ». Cependant, JA ne précise pas ce cadre émergentiste. Nous nous positionnerons, ici, dans la perspective d'un émergentisme linguistique (O'Grady, 2010; MacWhinney, 1998; entre autres) qui traite de propriétés " émergentistes » allant au-delà de la simple émergence traditionnellement définie comme l'apparition d'un élément $\mathrm{x}$. Cette vision du processus d'émergence est notamment apparue, pour la première fois, dans les travaux de John Stuart Mill (1843), philosophe, logicien et économiste britannique, qui propose que les systèmes complexes chimiques et physiques sont plus que la somme de leurs parties (O'Grady, 2010: 1). De ce fait, l'organisation et l'évolution des organismes complexes reposent sur leurs structures. L'action principale consiste, dès lors, à recomposer le système plutôt qu'à le décomposer (figure 1).

Figure 1 - Recomposition émergentiste des systèmes

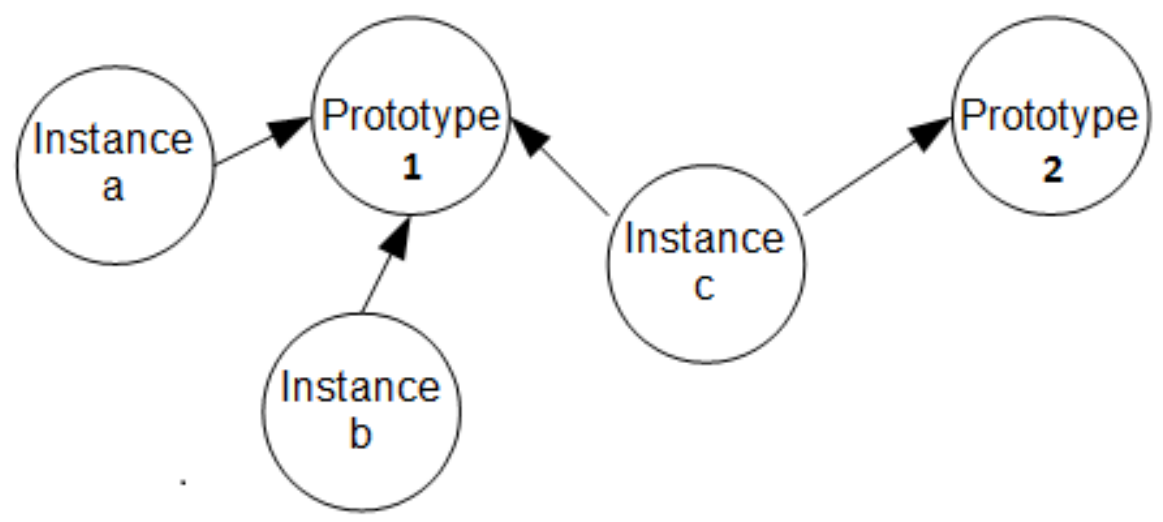

8 Les théories émergentistes reposent donc sur l'existence de propriétés des parties qui émergent, de manière différente, dans le système complexe qu'elles composent. Pour 
comprendre cette influence, il est indispensable de repérer deux types d'émergentisme (Stephan, 1999) :

- l'émergentisme faible (Idem: 1-2), proche du réductionnisme, fait l'état de propriétés émergentistes (certaines propriétés du système complexe ne se retrouvent pas dans les propriétés des parties) et des propriétés non-émergentistes (certaines propriétés du système se retrouvent dans celles des parties).

- l'émergentisme fort met en avant la notion d'irréductibilité (Idem: 2-5) (synchronic emergentism) : une propriété est émergentiste si et seulement si elle n'est pas réductible à la somme de propriétés des parties et la notion de nouveauté (diachronic emergentism) : il n'est pas possible de prédire le moment et le type de propriétés émergentistes qui apparaitront d'un tel système.

9 L'idée principale repose sur le postulat que ces phénomènes sont mieux expliqués s'ils font référence à des facteurs simples non-linguistiques et à leurs interactions (O'Grady et al., 1997 : 1). Ces éléments sont, de manière non-exhaustive, les propriétés physiologiques, perceptives, de la mémoire de travail, de la pragmatique, des interactions sociales, de l' input et des mécanismes d'apprentissage (O'Grady, 2010 : 2). Avant d'aller plus loin dans cette réflexion, il semble important d'ajouter que l'émergentisme ne récuse pas totalement la notion de nativisme en ce sens qu'il reconnaît l'existence de structures cérébrales innées, mais postule qu'il n'existe pas de structuration cérébrale codant des principes ou contraintes grammaticales (Jalilzadeh, 2011 : 153). Cela va dans le sens du neuroconstructivisme tel que le définit Karmiloff-Smith (1994). Nous pouvons donc dénombrer deux approches émergentistes pour l'acquisition des langues :

- «input-based emergentism " (O'Grady et al., 1997 : 2-4) : grandement basé sur le modèle de compétition (Mac Whinney, 1998) figurant que la fréquence du contact à un input langagier aura un impact majeur sur les processus développementaux et acquisitionnels.

- "processor-based emergentism» (Idem : 5-6) : théorisant que l'existence des composantes du langage (syntaxe, phonologie, etc.) est en réalité un ensemble de processus visant un traitement neurocognitif simple, économique et efficace.

10 Cette vision des éléments linguistiques, comme processus visant à réduire le poids du traitement neurocognitif et de la mémorisation, est au centre de l'émergentisme linguistique. Ainsi, la syntaxe est considérée comme émergeant de la conversation afin de faciliter le traitement sémantique et pragmatique; la morphologie s'opère face aux régularités statistiques en contact; le lexique est déterminé par la manière dont le cerveau stocke et gère les expériences, mais aussi face à la fréquence de mise en contact avec une occurrence (cette vision défie la notion de «mot» et de «phrase ») ; enfin la phonétique émerge en réponse aux différentes contraintes physiques des organes phonatoires et auditifs et la phonologie est un système d'actualisation des formes sonores en fonction de l'expérience (O'Grady, 2010 : 4-6).

11 Même si la relation corps-esprit n'est pas théorisée dans la littérature émergentiste actuelle, on peut en dessiner les contours et acceptions. Ellis (1998: 638) nous rappelle que "les interactions qui se déroulent à tous les niveaux, des gènes à l'environnement donne naissance à des formes et comportements émergents ». Medina (2000: 321$)$ va dans le même sens en disant que « la somme totale du cerveau et des interactions avec le corps forment l'idée de la conscience et de l'identité comme un tout ». Cette vision des liens corps-esprit, esquissés dans l'émergentisme, se retrouvent dans la théorie de la cognition incarnée. Pour Lakoff (2013), toute cognition est basée sur la connaissance qui vient de notre corps et les autres domaines sont cartographiés à partir de notre connaissance 
incarnée. Nous noterons que les opposants à la cognition incarnée suggèrent que les résultats empiriques qui soutiennent cette théorie peuvent aussi soutenir une vision désincarnée de la cognition (Mahon \& Caramazza, 2008). Goldman (2012), quant à lui, propose que cette approche, bien que partiellement non démontrée, peut être un déclencheur de rupture épistémologique des sciences. Il nous reste à penser une application de l'approche suggérée par JA à la phonétique-phonologie appliquée à la didactique des langues.

\section{Le primat de la perception : le cas de la didactique de la prononciation}

Pour JA, « il est impossible de séparer l'histoire de nos actions - biologiques et sociales de la façon dont le monde nous apparait ». Ce positionnement va dans le sens d'une perspective émergentiste linguistique dans laquelle la phonétique émergerait en réponse aux différentes contraintes physiques des organes phonatoires et auditifs et la phonologie serait un système d'actualisation des formes sonores en fonction de l'expérience. Nous nous positionnons, dès lors, en dehors d'une perspective traditionnelle considérant les organes de la phonation comme simple "machine à son" (Aubin, 1996: 37). Dans une approche bayésienne de la perception ${ }^{2}$, Ernst et Bülthoff $(2004: 164)$ affirment que notre perception du monde s'effectue dans une intentionnalité d'action et que nos actions affectent notre perception du monde environnant. Afin de permettre aux interactions d'avoir lieu, nous devons reconstruire notre environnement à partir de l'ensemble des modalités sensorielles cartographiées par le cerveau. Néanmoins, il est impossible d'enclencher ce processus par le seul traitement ascendant de l'information. De manière générale, les connaissances a priori (prior knowledge) sont les expériences passées reliées à un ou plusieurs média d'input sensoriel qui ont modifié le traitement neuronal de ces médias à long terme (Bransford et al, 2002: 14-15). Dans ce cadre bayésien, elles sont l'ensemble des structures neuronales (pré-) établies qui permettent de traiter l'input sensoriel ; ces structures sont, d'une certaine manière, déjà en place chez le nouveau-né à travers l'organisation neurophysiologique de l'embryon et l'expérience intra-utérine. Les connaissances a priori (prior knowledge) sont indispensables pour donner du sens à cette reconstruction. Les inférences bayésiennes nous permettent de modéliser un tel lien. Cette vision de l'expérience sensorielle fait écho, en psychocognition, au rôle de la mémoire de travail ${ }^{3}$ (Baddeley et Hitch, 1974) qui :

à l'interface entre la cognition et la mémoire à long terme, et l'administrateur central permettrait à l'individu d'organiser son comportement non pas en fonction de stimulations immédiates mais en fonction de représentations internes de l'environnement (Ollat, 1999 : 43).

13 Tiberghien (1999: 255) rappelle que « la mémoire ne concerne pas seulement le passé, elle détermine largement ce que sera notre présent perceptif. La mémoire génère en outre, de façon permanente, des schémas, des cadres d'interprétation qui façonnent nos anticipations. ». Nous noterons, cependant, que dans une perspective non-symbolique, la mémoire ne peut plus être considérée comme une "zone stable de stockage » mais comme un processus qui prend la forme des réseaux stabilisés. Pour Nadel et al. (2012:1), "la formation de la mémoire est un processus extrêmement dynamique ». Ce positionnement est prolongé par Squire (2004: 93) qui rappelle que « [c'est] une mémoire profondément différente... engagée dans l'action, ancrée dans le présent et regardant 
vers le futur... En vérité, elle ne représente plus notre passé, elle l'acte, et si elle mérite toujours le nom de mémoire, ce n'est pas parce qu'elle conserve des images révolues, mais parce qu'elle prolonge leurs effets pertinents dans le moment présent ».

Une autre manière de concevoir ce rapport corps-action-perception est la prise en compte des neurones miroirs (Rizzolatti \& Sinigaglia, 2006) qui constitueraient un ensemble de neurones s'activant que l'on fasse une action ou que l'on regarde une personne faire la même action. Les principales critiques des neurones miroirs sont repris par Hickok (2009) qui met en question la transférabilité du protocole expérimental du singe vers l'homme, le fait que l'activité des neurones miroirs pourrait refléter simplement un couplage sensori-moteur ou encore que la connaissance motrice pourrait influencer la compréhension de l'action jusqu'à un certain degré mais sans forcément soutenir la théorie selon laquelle la compréhension de l'action serait dépendante du système moteur.

Certains phénomènes, allant dans le sens de cette perspective, sont déjà connus mais leur implication sur les phénomènes de perception-production des sons notamment dans le processus d'enseignement/apprentissage a eu un impact épistémologique mineur en didactique des langues. Les manuels portant sur la didactique de la prononciation du français comme langue étrangère/seconde partent souvent $\mathrm{du}$ postulat qu'il faut percevoir auditivement pour produire articulatoirement, et ce, de manière unidirectionnelle. L'effet McGurk (McGurk \& Macdonald, 1976) soutient l'idée que la représentation phonologique des sons, dans une interaction humaine, dépend à la fois de la structure acoustique des sons mais aussi de signaux visuels (mouvements des lèvres). Ainsi un input labial de [ga] associé à un input auditif de [ba] se traduira par une perception phonologique de /da/. La théorie motrice de la perception ${ }^{4}$ (Libermann et Mattingly, 1985) suit le même principe en affirmant que les individus perçoivent les unités sonores en identifiant les gestes articulatoires par lesquels elles sont prononcées plutôt qu'en identifiant les schémas acoustiques générés par la parole.

Les travaux de Fonagy (1983) sur la psychophonétique tendent à démontrer le rapport entre les articulations de la parole et des représentations émotionnelles (Figure 2Figure). La contraction des muscles pharyngés accompagne la nausée, la pharyngalisation sera interprétée comme une expression de dégoût ou de déplaisir, voire de haine. La langue se rapprochant du palais peut représenter des objets petits : la palatalisation des sons est souvent associée dans les langues à la formation des diminutifs et elle est utilisée pour simuler un langage enfantin. L'arrondissement des lèvres chez la mère suggère la promesse d'un baiser, et une labialisation pourra servir comme indice d'un sentiment de tendresse (Vaissière, 2008). 


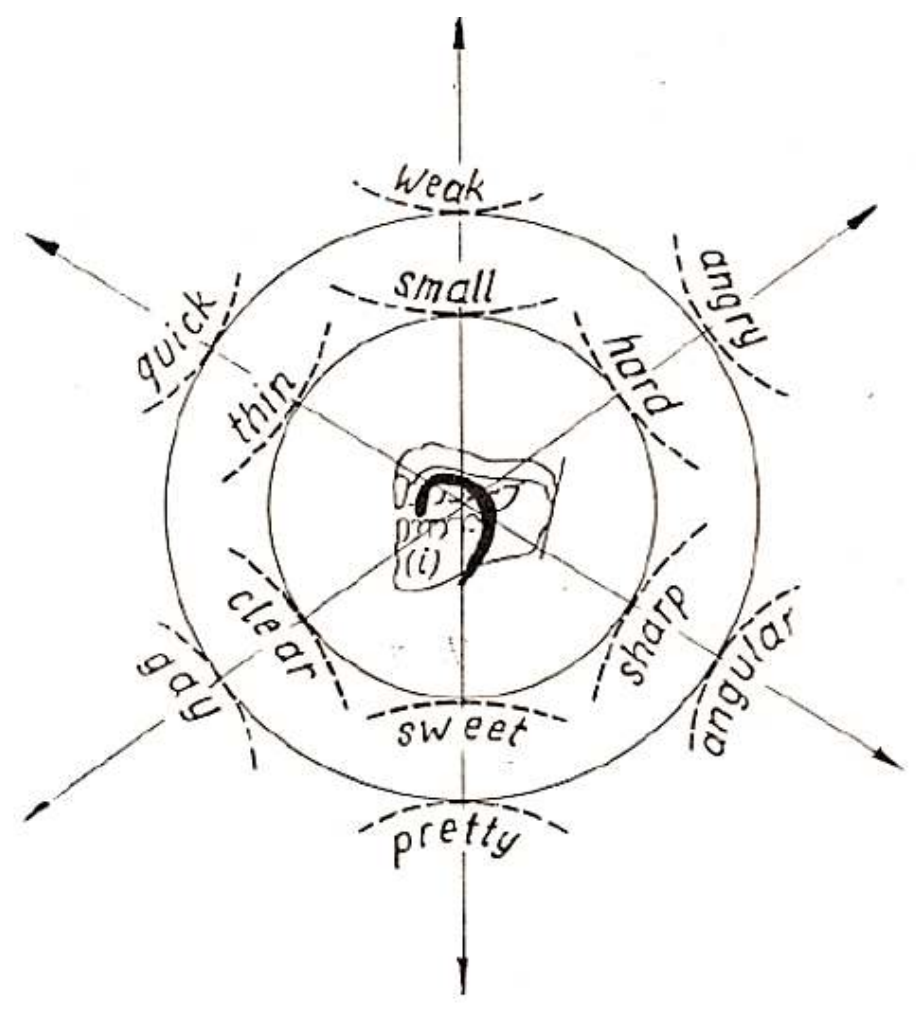

17 Cette vision fait écho à celle que rapporte Dortier (2012/2014 : 41 inspiré par Varela et al., 1991) pour qui «odeur, couleur, taille, goût et dégoût, les idées morales ont une indiscutable inscription corporelle et perceptive». On peut donc dire à travers cette analyse épistémologique préliminaire que même si l'émergentisme ne théorise pas totalement le corps dans l'émergence des caractéristiques de la parole, on peut voir que les traits linguistiques émergent de corps physiologiques, cognitifs et sociaux. La proposition de JA « de trouver de nouveaux outils de collectes et d'analyse des données », notamment pour la recherche en didactique des langues, prend tout son sens ici. Quels outils utiliser pour analyser les apports et limites de dispositifs didactiques qui s'inscrivent dans ce que Rolland (2011: 63) appelle le «kaléidoscope didactique humaniste » ? Ce dernier intègre des propositions, en didactique du FLE/S, qui vont dans le sens d'une meilleure intégration de la corporéité dans le processus de développement langagier. Renard (2002) rappelle que le «crible » ne se limite pas à celui phonologique et que ce dernier n'est que la partie visible d'un iceberg comprenant une part kinésique, proxémique, stylistique et dialectique (figure 3). Nous repréciserons ici que, dans notre position non-symbolique, la métaphore de «crible » est remise en question au profit de celle d'assimilation ${ }^{5}$ phonologique qui représente mieux l'idée d'un processus plutôt que celle d'un filtre à sons. De plus, l'approche de Renard permet d'appréhender la complexité des différents niveaux de cribles sans en préciser les liens ni les relations. On pourrait notamment se questionner sur la légitimité de décomposer ces éléments qui fonctionnent de manière interdépendante (voire émergente). 
Figure 3 - Les différents niveaux de cribles (Renard, 2002 : 229)

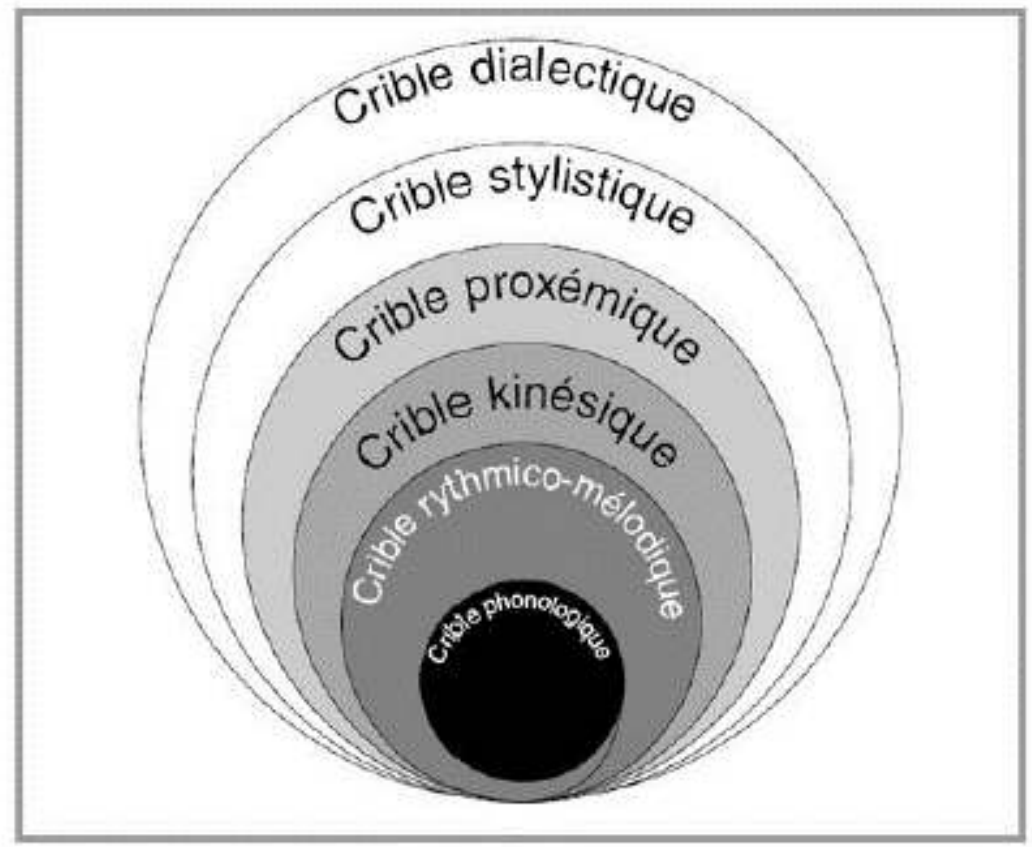

Dans ce sens, Guimbretière \& Kaneman-Pougatch (1991) ont proposé dans Le Plaisir des Sons, une intégration corporelle des sons et plus particulièrement ceux vocaliques. Même si les principes de cartographie des sons dans le corps peuvent être contestés ; peu ou pas d'études ont questionné l'impact cognitif d'une association entre les caractéristiques des sons de la parole et le corps dans son intégralité. 


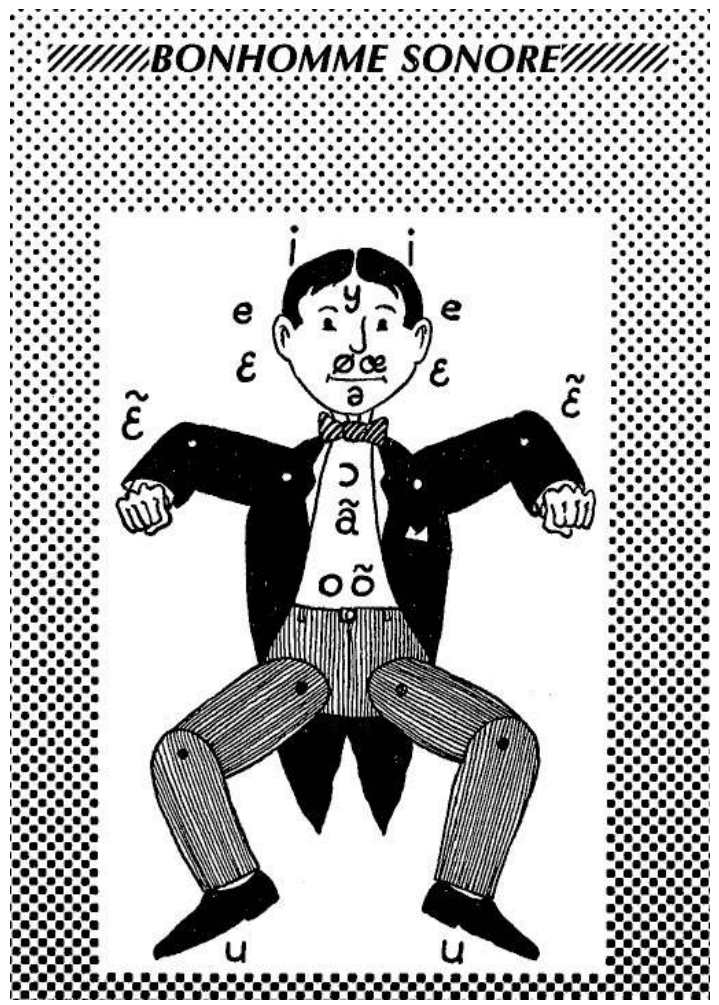

Cette tentative de questionner le positionnement de JA, à la lumière de la didactique de la prononciation $d u$ français langue étrangère/seconde, montre qu'il existe des cadres théoriques et des pratiques pédagogiques qui vont dans le sens d'une meilleure reconnaissance de l'expérience à la première personne. Cependant, force est de constater que peu d'études ont testé, de manière empirique, les apports et limites de ces propositions pédagogiques n'étant pas reconnues dans une épistémè scientifique contemporaine et/ou par la « didactique institutionnelle » (Rolland, 2011:50).

\section{Conclusion et perspectives}

Quel que soit le positionnement épistémologique dans lequel on se place, il est possible de mesurer les effets sur le développement langagier des liens corps-esprit. Le caractère « mou » qui est traditionnellement attribué aux sciences humaines et sociales ne doit pas faire oublier que dans d'autres domaines où les sciences sont dites exactes, on modélise des objets dont on ne peut mesurer que les effets et non les caractéristiques tangibles (cf. la matière noire; Milgrom, 2002). Dans About Consciousness \& Emergentism, Medina (2000 : 323) rappelle qu' :

il y a de nombreux vides à combler, et c'est vrai pour tout en sciences, plus particulièrement dans le domaine des liens entre les gènes et le comportement [...] Il est important pour toutes et tous de se rappeler qu'ils travaillent à partir d'une connaissance incomplète, peu importe ô combien ils croient dans les données empiriques actuelles.

Rolland (2011: 118) souligne que « la nature même de la didactique des langues est de ne pas se focaliser sur un seul paradigme, par souci d'éviter l'enfermement dans une seule école de pensée ». Une pédagogie des langues, et plus particulièrement celle de la 
prononciation, qui prendrait en compte « une philosophie de l'être comme expérience et de l'expérience comme être qui s'inscrit dans une épistémologie relationnelle et de la conscience enactée du monde » (PA) existe déjà. Un certain nombre de pédagogues intègre cette dimension dans les pratiques sur le terrain, mais il reste, comme le mentionne Joëlle Aden, à déterminer des outils scientifiques didactiques qui permettront d'analyser au mieux les effets sur le développement langagier. Cependant, nous nous questionnons sur le fait que pour atteindre ce but, plutôt que de chercher de nouveaux outils et méthodes comme le propose JA, il conviendrait de renforcer le poids des équipes pluridisciplinaires dont la complémentarité serait forgée autour d'une volonté de travailler ensemble avant de vouloir trouver un consensus scientifique initial. On peut cependant penser qu'un consensus émergera au fur et à mesure d'un tel projet.

Pour terminer, rappelons qu'un dispositif sans corps est une "classe » sans individuapprenant et une recherche sans individu-chercheur. L'individualisation des formations passe par une meilleure prise compte de l'individu dans sa globalité ce qui inclus des corps. Plus qu'un changement brutal de paradigme, cette réflexion invite les individus, qu'ils-elles soient enseignant-e-s, chercheur-euse-s, apprenant-e-s ou les trois en même temps, à être ouvert à la pluralité des points de vue et aux intersections entre les positionnements. Frith $(2010: 153)$ nous y invite quand il affirme que «notre perception du monde est un fantasme qui coïncide avec la réalité ».

\section{BIBLIOGRAPHIE}

Aubin, S. (1996). La didactique de la musique du français : sa légitimité, son interdisciplinarité. Villeneuve d'Ascq : ANRT. Thèse de doctorat soutenue à Rouen.

Baddeley, A. \& Hitch, G.J. (1974). « Working Memory ». In Bower, G. Eds. Recent Advances in learning and motivation. Volume 8. New York: Academic Press. pp. 47-90.

Bransford, J.D., Brown, A.L., \& Cocking, R.R. Eds. (2002). How people learn : Brain, mind, experience, and school. Washington, D.C.: National Academy Press.

Block, D. (1999). « Who framed SLA research?». In Cameron, Lynne \& Graham Low, Researching and Applying Metaphor. Cambridge : Cambridge University Press.

Bourdieu, P. (1979). La distinction. Paris : Minuit.

Claverie, B. (2010). «Pluri-, inter-, transdisciplinarité : ou le réel décomposé en réseaux de savoir ». Projectics/Proyéctica/Projectique. $\mathrm{N}^{\circ}$ 4. pp. 1-14.

Courville, D. (2009). «L'ambiguïté du corps chez Husserl : entre une égologie désincarnée et une phénoménologie de la chair ». Revue PHARES. vol. 9. pp. 62-79.

Demars, L.-P. (2009). « La distinction corps-âme est-elle encore valable aujourd'hui ? Une critique du dualisme cartésien ». Revue PHARES. vol. 9. pp. 9-31.

Dortier, J.-F. (2012/2014). Le cerveau et la pensée : le nouvel âge des sciences cognitives. Paris: Editions Sciences Humaines.

Recherches en didactique des langues et des cultures, 14-1 | 2017 
Ellis, N. C. (1998). « Emergentism, connectionism and language learning ». Language Learning. 48: 4. pp. 631-64.

Ernst, M.O. \& Bülthoff, H. (2004). « Merging the senses into a robust percept ». Trends in cognitive Sciences. Volume 8, $\mathrm{N}^{\circ} 4$. pp 162-9.

Fonagy, I. (1983). La vive voix : Essais de psycho-phonétique. Paris : Payot.

Frith, C. (2010). Comment le cerveau crée notre univers mental. Paris: Odile Jacob.

Goldman, A. I. (2012). « A moderate approach to embodied cognitive science ». Review of Philosophy and Psychology. vol. 3, Issue 1. pp. 71-88.

Guimbretière, E \& Kaneman-Pougatch, M. (1991). Plaisir des sons : Enseignement des sons du français. Paris: Les Éditions Didier.

Halle, M. (2002). From Memory to Speech and Back: Papers on phonetics and phonology 1954-2002. New York NY: Mouton de Gruyter.

Hickok, G. (2009). « Eight problems for the mirror neuron theory of action understanding in monkeys and humans ». J. Cogn Neurosci. 21(7). pp. 1229-1243.

Hofstadter, D. \& Sander, E. (2013). L'analogie. Cœur de la pensée. Paris: Odile Jacob.

Jalilzadeh, K. (2011). « Emergentism in First Language Acquisition ». European Journal of Scientific Research. Volume 57, $\mathrm{N}^{\circ}$ 1. pp. 152-155.

Karmiloff-Smith, A. (1994). « Précis of Beyond Modularity: A Developmental Perspective on Cognitive Science ». Behavioral and Brain Sciences. $N^{\circ} 17$. pp. 693-745.

Lakoff, G. (2013). « Embodied cognition and language ». Lecture given on October 22, 2013at Central European University in Budapest, Hungary. Lien : https://www.youtube.com/watch ?

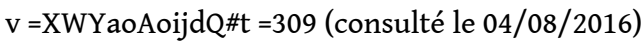

Liberman, A. \& Mattingly, I. (1985). « The motor theory of speech perception revised ». Cognition. Volume 21. pp. 1-36.

Loty, L. (2005). « Pour l'indisciplinarité », The Interdisciplinary Century; Tensions and convergences in 18th-century Art, History and Literature, edited by Julia Douthwaite and Mary Vidal, Oxford, Studies on Voltaire and the Eighteenth Century, 04, Voltaire Foundation. pp. 245-259.

MacWhinney, B. (1998). « Models of the emergence of language ». Annual Review of psychology. N -49. pp. 199-227.

Mahon, B. Z. \& Caramazza, A. (2008). « A critical look at the embodied cognition hypothessis and a new proposal for grounding conceptual content ». Journal of Physiology. 102. pp. 59-70.

McGurk, H. \& MacDonald, J. (1976). « Earing lips and seeing voices ». Nature. 264. pp. 746-748.

Medina, J. (2000). The genetic inferno: inside the seven deadly sins. Cambridge: Cambridge University Press.

Mill, J. S. (1843/2002). A system of Logic, Ratiocinative and inductive. Honolulu: University Press of the Pacitic.

Milgrom, M. (2002). « Does dark matter really exist », Scientific American. pp. 42-52.

Morin, E. (1990). « Carrefour des sciences », Actes du Colloque du Comité National de la Recherche Scientifique Interdisciplinarité, Éditions du CNRS.

Morin, E., \& Lemoigne, J.-L. (1999). Introduction à la pensée complexe. Paris: L'Harmattan. 
Nadel, L., Hupbach, A., Gomez, R. \& Newman-Smith, K. (2012). « Memory formation, consolidation and transformation ». Neuroscience and Biobehavioral Reviews. 36. pp. 1640-45.

Narcy-Combes, J.-P. (2014). « De la complémentarité des domaines pour un didacticien des langues : quelle place pour les multilittéracies? ». FLuL (Fremdsprachen Lehren und Lernen). 43(2). pp. $29-42$

Narcy-Combes, J.-P. \& Narcy-Combes, M.-F. (2000). « Épistémologie et méthodologie de la recherche dans le secteur LANSAD : qu'apporterait une harmonisation des pratiques ? ", ASp, 27-30. pp. 243-251.

O'Grady, W. Lee, M. \& Kwak, H.-Y. (1997). « Emergentism and Second Language Acquisition ». In Ritchie, W. \& Bhatia, T. Eds. The handbook of Second Language Acquisition. Second edition. pp. 69-88.

O'Grady, W. (2010). « Emergentism ». Cambridge Encyclopedia of Language Science. http:// www.ling.hawaii.edu/faculty/ogrady/Emergentism.pdf (consulté le 22/09/11)

Ollat, H. (1999). « Schizophrénie et mémoire de travail ». Neuropsychiatrie : Tendances et Débats. 4. pp. 43-50.

Piaget, J. (1964). Six études de psychologie. Paris : Folio.

Piaget, J. (1968). Le structuralisme. Paris : PUF. 12ième édition, $n^{\circ} 1311$.

Randall, M. (2007). Memory, Psychology and Second Language Learning. Amsterdam : John Benjamins.

Renard, R. (2002). Apprentissage d'une langue étrangère-seconde. 2, La phonétique verbo-tonale.

Bruxelles : De Boeck Université.

Rolland, Y. (2011). Apprendre à prononcer. Quels paradigmes en didactique des langues? Paris : Belin.

Rizzolatti, G. \& Sinigaglia, C. (2006). Les neurones miroirs. Paris : Odile Jacob.

Seron, X. (1998). « Le programme de la neuropsychologie ». In Seron, X. \& Jeannerod, M. (1998). Neurophsychologie humaine. Paris : Mardaga. pp. 35-56

Simard, R. (2009). « Eloge du tiers toujours exclu : reconsidérer le monisme de l'esprit ». Revue PHARES. vol. 9. pp. 32-61.

Stephan, A. (1999). « Varieties of emergentism ». Evolution and Cognition. Volume 5, № 1. pp. 49-59.

Tiberghien, G. (1999). « Chapitre 9 La mémoire Partie 1 : Psychologique cognitive de la mémoire humaine ». In Seron, X. \& Jeannerod, M. (1999). Neuropsychologie humaine. Paris : Mardaga.

Vaissière, J. (2008). « Ivan Fonagy ; un pionnier dans l'étude de l'expressivité dans la parole ». Conférence donnée dans le cadre du Festival Agora de l'Ircam. 5-20 Juin.

Varela, F., Thompson, E. \& Rosch, E. (1991). The embodied mind: Cognitive science and human experience. Cambridge, MA : MIT Press.

\section{NOTES}

1. "L'assimilation suppose que ce qui est perçu est intégré dans notre monde interne sans en changer sa structure, mais avec le risque de mauvaises classifications internes » (Rolland, 2011 : $31)$.

2. La théorie bayésienne est une vision probabiliste permettant de théoriser certains fonctionnements cognitifs dont la perception. Elle est basée sur le théorème de Thomas Bayes qui repose sur le principe qu' «étant donné un certain phénomène A que nous souhaitons connaître 
et une observation $\mathrm{X}$ se rapportant à $\mathrm{A}$, le théorème de Bayes nous dit de combien nous devrions actualiser la connaissance de A en fonction de la nouvelle observation X » (Frith, 2010 : 165-166).

3. La mémoire de travail, selon Randall (2007: 27), se base sur des principes connexionnistes et des concepts associatifs.

4. Théorie dont les principes ont reçu l'aval de Halle (2002) selon Randall (2007:45).

5. Telle que présentée par Piaget (1964).

\section{RÉSUMÉS}

L'article de Joëlle Aden envisage la mise en place d'un paradigme enactif qui participerait à définir les contours d'une méthode d'exploration de l'expérience en $1^{\text {ère }}$ personne. Nous tenterons, ici, de questionner ce cadre à travers une analyse de la place du corps dans l'émergentisme linguistique. Nous reviendrons notamment sur son analyse épistémologique qui l'a conduite à considérer le fait que nous sommes DANS le langage. Nous soutiendrons ses propositions d'un tel changement épistémologique dans le champ de l'éducation et dans la recherche en didactique des langues tout en prenant du recul sur la possibilité de généraliser un tel cadre.

In her paper, Joëlle Aden considers the implementation of an enactive approach which would participate in defining the contours of a first-person experienced method of exploration. We will try to question this framework by analyzing how the body is theorized in linguistic emergentism. We will reconsider her epistemological analysis which led her to acknowledge we are INSIDE language. We will follow her proposition for an epistemological shift in the field of education and applied linguistics while considering, with hindsight, the possibility of generalizing such a framework.

\section{INDEX}

Mots-clés : corps, didactique des langues, émergentisme, disciplinarité, prononciation

Keywords : body, applied linguistics, emergentism, disciplinarity, pronunciation

\section{AUTEUR}

\section{GRÉGORY MIRAS}

Grégory Miras est maitre de conférences au laboratoire DYLIS à l'université de Rouen Normandie. Ses thématiques de recherche portent à la fois sur la compréhension des mécanismes de perception/production des sons en langue étrangère mais aussi sur les technologies numériques pour l'enseignement/apprentissage des langues.

Courriel : gy.miras[a]gmail.com.

Toile : https://sites.google.com/site/gregorymiras/home. 